\title{
ON MINIMALITY OF DETERMINANTAL VARIETIES
}

\author{
KHAZHGALI KOZHASOV
}

\begin{abstract}
We prove that semialgebraic sets of rectangular matrices of a fixed rank, of skew-symmetric matrices of a fixed rank and of real symmetric matrices whose eigenvalues have prescribed multiplicities are minimal submanifolds of the space of real matrices of a given size.
\end{abstract}

\section{INTRODUCTION}

Minimal surfaces are mathematical abstractions of soap films. They are surfaces in $\mathbb{R}^{3}$ that locally minimize the area. In 1760s Lagrange posed the problem of finding the surface of least area among all surfaces in $\mathbb{R}^{3}$ with a given closed boundary curve. Necessarily, a solution to this problem must be a minimal surface. It was shown by Monge in 1776 that a surface is minimal if and only if its mean curvature is zero everywhere. It is also of importance to look at higher-dimensional analogs of minimal surfaces. These are submanifolds of an Euclidean space (or, more generally, of a Riemannian manifold) with zero mean curvature vector field, see Subsection 2.4 for details. A nice account and survey of results about minimal surfaces and minimal submanifolds can be found in [TF87].

Even though examples of minimal submanifolds are abound, there are not so many of those that are defined by algebraic equations [Hsi67].

In [Tka10] Tkachev showed that the smooth locus $\mathscr{M}_{n, n, n-1}$ of the affine variety of singular real matrices of size $n \times n$ is a minimal hypersurface in the Euclidean space of all $n \times n$ matrices. To prove his result Tkachev shows that the determinant of a square matrix is an eigenfunction of the mean curvature operator, a condition known to be equivalent to the minimality of the associated hypersurface $\mathscr{M}_{n, n, n-1} \subset\{$ det $=0\}$. In Theorem 1.1 we extend Tkachev's result to the semialgebraic submanifold $\mathscr{M}_{m, n, r}$ of $m \times n$ real matrices of rank $r$ and prove its minimality using a local parametrization of $\mathscr{M}_{m, n, r}$ coming from singular value decomposition (2.2).

In [HLT17] Hoppe, Linardopoulos and Turgut proved that the smooth locus $\delta k_{2 n, 2 n-2}$ of the affine variety of singular real skew-symmetric matrices of size $2 n \times 2 n$ is a minimal hypersurface in the Euclidean space of all $2 n \times 2 n$ skew-symmetric matrices. The authors of [HLT17] show that the pfaffian of a skew-symmetric matrix of an even size is an eigenfunction of the mean curvature operator thus proving minimality of the hypersurface $\delta k_{2 n, 2 n-2} \subset\{\mathrm{pf}=0\}$. In Theorem 1.3 we extend the result of Hoppe et al.

2010 Mathematics Subject Classification. 14P05, 14M12, 15A03, 15A15, 15A18, 49Q05, 53A10.

Key words and phrases. Determinantal varieties, minimal submanifolds, singular value decomposition, symmetric matrices with repeated eigenvalues. 
to the semialgebraic submanifold $\delta k_{n, 2 r}$ of $n \times n$ real matrices of rank $2 r$ and prove its minimality using a local parametrization of $\delta k_{n, 2 r}$ coming from the normal form (2.4) of a skew-symmetric matrix.

Finally, in Theorem 1.6 we prove minimality of the set of real symmetric matrices whose eigenvalues have prescribed multiplicities.

\section{MAIN RESULTS}

For $m \leq n$ let $\mathscr{M}_{m, n}$ denote the space of $m \times n$ real matrices endowed with the Frobenius inner product

$$
\langle A, B\rangle=\operatorname{Tr}\left(A^{t} B\right)=\sum_{i=1}^{m} \sum_{j=1}^{n} a_{i j} b_{i j}, \quad A=\left(a_{i j}\right), B=\left(b_{i j}\right) \in \mathscr{M}_{m, n} .
$$

For a fixed $r \leq m \leq n$ denote by

$$
\mathscr{M}_{m, n, r}=\left\{A \in \mathscr{M}_{m, n}: \operatorname{rank}(A)=r\right\}
$$

the semialgebraic set of $m \times n$ matrices of rank $r$. It is well-known that $\mathscr{M}_{m, n, r}$ is the smooth locus of the affine variety

$$
\overline{\mathscr{M}}_{m, n, r}=\left\{A \in \mathscr{M}_{m, n}: \operatorname{rank}(A) \leq r\right\}
$$

of $m \times n$ matrices of rank at most $r$.

If $m=n$ and $r=n-1$ the variety

$$
\overline{\mathscr{M}}_{n, n, n-1}=\left\{A \in \mathscr{M}_{n, n}: \operatorname{det}(A)=0\right\}
$$

of singular $n \times n$ matrices is a hypersurface in $\mathscr{M}_{n, n}$. In Tka10] Tkachev proved that the smooth semialgebraic hypersurface $\mathscr{M}_{n, n, n-1}$ is minimal in $\left(\mathscr{M}_{n, n},\langle, \cdot, \cdot\rangle\right)$. A submanifold of a Riemannian manifold is said to be minimal if its mean curvature vector field is identically zero, see Subsection 2.4 for details. In our first main result we generalize this result to all determinantal submanifolds $\mathscr{M}_{m, n, r}$.

Theorem 1.1. For $r \leq m \leq n$ the smooth semialgebraic set $\mathscr{M}_{m, n, r}$ is a minimal submanifold of $\left(\mathscr{M}_{m, n},\langle\cdot, \cdot\rangle\right)$.

We give a proof of Theorem 1.1 in Subsection 3.1. Our proof generalizes the proof of minimality of the 4-dimensional submanifold $\mathscr{M}_{2,3,1} \subset \mathscr{M}_{2,3}$ of $2 \times 3$ matrices of rank one given in [Hop19, p. 37].

One can also consider the projective semialgebraic set $\mathbb{P}\left(\mathscr{M}_{m, n, r}\right) \subset \mathbb{P}\left(\mathscr{M}_{m, n}\right)$ of $m \times n$ real matrices of rank $r$. For this one endows the real projective space $\mathbb{P}\left(\mathscr{M}_{m, n}\right)$ with the standard metric induced from (1.1), see Subsection 2.5. The following corollary is then implied by Theorem 1.1 and Proposition 2.6.

Corollary 1.2. For $r \leq m \leq n$ the smooth projective semialgebraic set $\mathbb{P}\left(\mathscr{M}_{m, n, r}\right)$ is a minimal submanifold of $\mathbb{P}\left(\mathscr{M}_{m, n}\right)$.

Let us denote by

$$
\delta \aleph_{n}=\left\{A=\left(a_{i j}\right) \in \mathscr{M}_{n, n}: a_{i j}=-a_{j i} \text { for any } i, j\right\}
$$


the space of $n \times n$ real skew-symmetric matrices. The rank of a skew-symmetric matrix is even and, in particular, any skew-symmetric matrix of an odd size is singular. In the following, for $1 \leq r \leq\lfloor n / 2\rfloor$ let

$$
\delta k_{n, 2 r}=\delta k_{n} \cap \mathscr{M}_{n, n, 2 r}=\left\{A \in \delta k_{n}: \operatorname{rank}(A)=2 r\right\}
$$

denote the semialgebraic set of $n \times n$ skew-symmetric matrices of rank $2 r$. It is well-known that $\delta k_{n, 2 r}$ is the smooth locus of the skew-symmetric determinantal variety

$$
\bar{\delta}_{n, 2 r}=\left\{A \in \delta \hbar_{n}: \operatorname{rank}(A) \leq 2 r\right\}
$$

of $n \times n$ skew-symmetric matrices of rank at most $2 r$. If $r=n-1$ the variety

$$
\overline{\delta k}_{2 n, 2 n-2}=\left\{A \in \delta k_{2 n}: \operatorname{det}(A)=0\right\}
$$

of singular skew-symmetric matrices is a hypersurface in $\delta k_{2 n}$ cut out by the pfaffian polynomial that is defined via $\operatorname{pf}(A)=\operatorname{det}(A)^{2}, A \in \delta k_{2 n}$. In [HLT17] it was discovered that $\delta k_{2 n, 2 n-2}$ is a minimal hypersurface in $\delta k_{2 n}$. In our second main result we generalize this fact to all skew-symmetric determinantal submanifolds $\delta k_{n, 2 r}$.

Theorem 1.3. For $1 \leq r \leq\lfloor n / 2\rfloor$ the smooth semialgebraic set $\delta_{k_{n, 2 r}}$ is a minimal submanifold of $\left(\delta k_{n},\langle\cdot, \cdot\rangle\right)$.

We give a proof of Theorem 1.3 in Subsection 3.2 .

One can also consider the projective semialgebraic set $\mathbb{P}\left(\delta k_{n, 2 r}\right) \subset \mathbb{P}\left(\delta k_{n}\right)$ of $n \times n$ real skew-symmetric matrices of rank $r$. One again endows the real projective space $\mathbb{P}\left(\delta k_{n}\right)$ with the standard metric induced from (1.1), see Subsection 2.5. The following corollary is then implied by Theorem 1.3 and Proposition 2.6.

Corollary 1.4. For $1 \leq r \leq\lfloor n / 2\rfloor$ the smooth projective semialgebraic set $\mathbb{P}\left(\mathcal{S} k_{n, 2 r}\right)$ is a minimal submanifold of $\mathbb{P}\left(\mathcal{S} \ell_{n}\right)$.

Let us denote by

$$
\operatorname{sym}_{n}=\left\{A=\left(a_{i j}\right) \in \mathscr{M}_{n, n}: a_{i j}=a_{j i} \text { for any } i, j\right\}
$$

the space of $n \times n$ real symmetric matrices.

Remark 1.5. Theorems 1.1 and 1.3 may suggest that the symmetric determinantal submanifold $\mathscr{M}_{n, n, r} \cap$ Sym $_{n}$ of symmetric matrices of rank $r$ is minimal in $\left(\right.$ Sym $\left._{n},\langle\cdot, \cdot\rangle\right)$. However, it is not in general the case. For example, it is easy to see that the surface

$$
\left\{a=\left(\begin{array}{ll}
a_{11} & a_{12} \\
a_{12} & a_{22}
\end{array}\right): \operatorname{det}(a)=a_{11} a_{22}-a_{12}^{2}=0\right\} \subset \text { sym }_{2}=\mathbb{R}^{3}
$$

of singular $2 \times 2$ real symmetric matrices has non-zero mean curvature.

Given a real symmetric matrix $A \in \delta$ Sym $_{n}$ let us denote by $\chi_{A}(t)=\operatorname{det}(t \mathrm{Id}-A)$ its characteristic polynomial. An eigenvalue $\lambda$ of $A \in$ Sym $_{n}$ has multiplicity $m$, where $1 \leq m \leq n$, if $\chi_{A}^{(i)}(\lambda)=0$ for $i=0, \ldots, m-1$ and $\chi_{A}^{(m)}(\lambda) \neq 0$.

For a vector $\vec{\kappa}=\left(\kappa_{1}, \kappa_{2}, \ldots\right)$ of non-negative integers such that $1 \kappa_{1}+2 \kappa_{2}+\cdots=n$ let us denote by

$$
\operatorname{Sym}_{n, \vec{\kappa}}=\left\{A \in \operatorname{Sym}_{n}: A \text { has } \kappa_{i} \text { eigenvalues of multiplicity } i\right\}
$$


the semialgebraic set of $n \times n$ real symmetric matrices that have $\kappa_{i}$ eigenvalues of multiplicity $i, i \geq 1$. Sets $\delta y m_{n, \vec{\kappa}}$ are smooth submanifolds of $\delta y m_{n}$ and they form a stratification of Sym $_{n}$ with $\mathcal{S y m}_{n,(n)}$ being the unique open stratum [Arn72]. They were studied in Arn72, Agr11, BKL18]. We discover a new fact about Sym ${ }_{n, \vec{\kappa}}$, namely its minimality.

Theorem 1.6. For any vector $\vec{\kappa}=\left(\kappa_{1}, \kappa_{2}, \ldots\right)$ the smooth semialgebraic set Sym ${ }_{n, \vec{\kappa}}$ is a minimal submanifold of $\left(\right.$ Sym $\left._{n},\langle\cdot, \cdot\rangle\right)$.

We give a proof of Theorem 1.6 in Subsection 3.3. One can again consider the projective version $\mathbb{P}\left(\mathcal{S y m}_{n, \vec{\kappa}}\right) \subset \mathbb{P}\left({\mathcal{S} y m_{n}}\right)$ consisting of $n \times n$ real symmetric matrices with $\kappa_{i}$ eigenvalues of multiplicity $i, i \geq 1$. The real projective space $\mathbb{P}\left({\mathcal{S} y m_{n}}_{n}\right)$ is endowed with the standard metric induced from (1.1), see Subsection 2.5. Theorem 1.6 and Proposition 2.6 imply the following corollary.

Corollary 1.7. For any vector $\vec{\kappa}=\left(\kappa_{1}, \kappa_{2}, \ldots\right)$ the smooth projective semialgebraic set $\mathbb{P}\left(\right.$ Sym $\left._{n, \vec{k}}\right)$ is a minimal submanifold of $\mathbb{P}\left(\right.$ Sym $\left._{n}\right)$.

\section{Preliminaries AND AUXILIARY RESUlts}

In this section we state some facts and results that we then use in Section 3 to prove our main results.

2.1. Singular value decomposition. Let $O(n)=\left\{V \in \mathscr{M}_{n, n}: V^{\top} V=\right.$ id $\}$ denote the group of orthogonal $n \times n$ matrices. The standard action of the product $O(m) \times O(n)$ of orthogonal groups on $\mathscr{M}_{m, n}$,

$$
(U, V) \in O(m) \times O(n), A \in \mathscr{M}_{m, n} \mapsto U A V^{\top},
$$

preserves the Frobenius inner product (1.1), that is, for $(U, V) \in O(m) \times O(n)$

$$
\left\langle U A V^{\top}, U B V^{\top}\right\rangle=\operatorname{Tr}\left(V A^{\top} U^{\top} U B V^{\top}\right)=\operatorname{Tr}\left(A^{\top} B\right)=\langle A, B\rangle, \quad A, B \in \mathscr{M}_{m, n} .
$$

Moreover, the action (2.1) obviously preserves the rank of a matrix and hence the manifold $\mathscr{M}_{m, n, r}$ is invariant under $O(m) \times O(n)$ for any $r \leq m \leq n$. The singular value decomposition (in the following SVD) of a matrix $A \in \mathscr{M}_{m, n}$ is a factorization

$$
A=U^{\top} \Sigma V
$$

where $U \in O(m), V \in O(n)$ and

$$
\Sigma=\left(\begin{array}{cccc}
\sigma_{1} & & 0 & \\
& \ddots & & 0 \\
0 & & \sigma_{m} &
\end{array}\right)
$$

is the "diagonal" matrix of singular values $\sigma_{1}, \ldots, \sigma_{m} \geq 0$ of $A$. Note that the number of non-zero singular values equals the rank of $A$ and we can, without loss of generality, assume that they are ordered, $\sigma_{1} \geq \cdots \geq \sigma_{m} \geq 0$.

In Subsection 3.1 we use the singular value decomposition (2.2) of a matrix in order to design a local parametrization of $\mathscr{M}_{m, n, r}$ suitable for computing its mean curvature. 
2.2. Normal form of a skew-symmetric matrix. Consider the following diagonal subaction of the action (2.1) on the space $\delta k_{n}$ of $n \times n$ real skew-symmetric matrices

$$
V \in O(n), A \in \delta k_{n} \mapsto V A V^{\top} .
$$

Any $A \in S k_{n}$ can be written in the normal form [Tho88, Thm. 2.5]

$$
A=V^{\top} \Omega V
$$

where $V \in O(n)$ and

$$
\Omega=\left(\begin{array}{cccccccc}
0 & \omega_{1} & & & & & & \\
-\omega_{1} & 0 & & & & 0 & & \\
& & \ddots & & & & & \\
& & & 0 & \omega_{r} & & & \\
& & & -\omega_{r} & 0 & & & \\
& & & & & 0 & & \\
& & & & & & \ddots & 0
\end{array}\right),
$$

where $\pm i \omega_{1}, \ldots, \pm i \omega_{r} \in i \cdot \mathbb{R}$ are non-zero eigenvalues of $A$ and, in particular, $2 r$ is the rank of $A$. We can, moreover, assume that $\omega_{1} \geq \cdots \geq \omega_{r}>0$ are positive and ordered.

In Subsection 3.2 we use the normal form (2.4) of a skew-symmetric matrix in order to design a local parametrization of $\delta k_{n, 2 r}$ suitable for computing its mean curvature.

2.3. Spectral decomposition of a symmetric matrix. Consider the following diagonal subaction of the action (2.1) on the space $\delta y m_{n}$ of $n \times n$ real symmetric matrices

$$
V \in O(n), A \in \operatorname{Sym}_{n} \mapsto V A V^{\top} .
$$

The spectral decomposition of a matrix $A \in \mathcal{S y m}_{n}$ is a factorization

$$
A=V^{\top} \Lambda V
$$

where $V \in O(n)$ and

$$
\Lambda=\left(\begin{array}{lll}
\lambda_{1} & & 0 \\
& \ddots & \\
0 & & \lambda_{n}
\end{array}\right)
$$

is the diagonal matrix of eigenvalues $\lambda_{1}, \ldots \lambda_{n} \in \mathbb{R}$ of $A$. Note that singular values of $A$ are related to its eigenvalues via $\sigma_{i}=\left|\lambda_{i}\right|, i=1, \ldots, n$.

Semialgebraic manifolds $\delta y m_{n, \vec{\kappa}}$ are invariant under the action (2.6). It is not difficult to show that the membership of $A \in \delta y m_{n}$ in a certain Sym $_{n, \kappa}$ is determined by the conjugacy class of its stabilizer under (2.6). To prove this, we need the following lemma.

Lemma 2.1. Let $A \in$ Sym $_{n, \vec{\kappa}}$. The stabilizer $O(n)_{A}$ of $A$ under the action (2.6) equals

$$
O(n)_{A}=V^{\top} O_{\vec{\kappa}} V,
$$


where $V \in O(n)$ and

$$
O_{\vec{\kappa}}=\chi_{i \geq 1} O(i)^{\kappa_{i}}=\chi_{i \geq 1} \underbrace{O(i) \times \cdots \times O(i)}_{\kappa_{i} \text { times }}
$$

is the direct product of orthogonal groups of sizes encoded by the partition $\vec{\kappa}$.

Proof. By (2.7) we can write $A=V^{\top} \Lambda V$ for some $V \in O(n)$ and diagonal matrix $\Lambda$ of eigenvalues. Since $A \in \mathcal{S y m}_{n, \vec{\kappa}}$ there are $|\vec{\kappa}|=\kappa_{1}+\kappa_{2}+\ldots$ pairwise distinct eigenvalues that we denote by $\lambda_{j}^{(i)}, i \geq 1, j=1, \ldots, \kappa_{i}$. After a possible permutation of the entries of $\Lambda$, we can assume that it is of the form

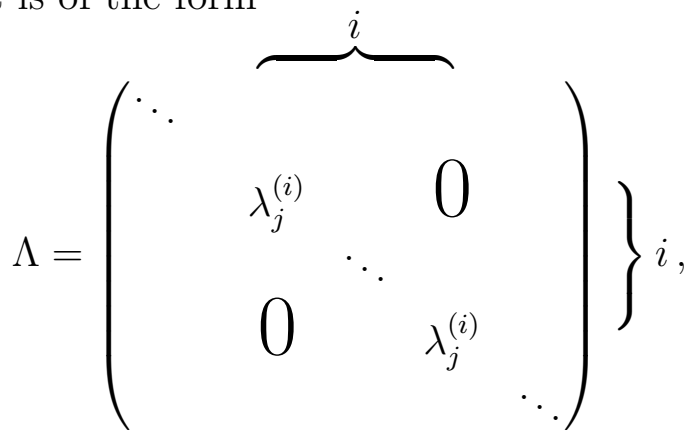

namely the diagonal entries of $\Lambda$ are $\lambda_{1}^{(1)}, \ldots, \lambda_{\kappa 1}^{(1)}, \lambda_{1}^{(2)}, \lambda_{1}^{(2)}, \ldots, \lambda_{\kappa_{2}}^{(2)}, \lambda_{\kappa_{2}}^{(2)}, \lambda_{1}^{(3)}, \lambda_{1}^{(3)}, \lambda_{1}^{(3)}, \ldots$

Let $U \in O(n)_{A}$ be an orthogonal matrix that fixes $A$, that is, $U V^{\top} \Lambda V U^{\top}=U A U^{\top}=$ $A=V^{\top} \Lambda V$ or, equivalently, $V U V^{\top} \Lambda=\Lambda V U V^{\top}$. Denoting $W=V U V^{\top}$ and taking the $(i, j)$ th entry of $W \Lambda=\Lambda W$ we obtain

$$
W_{i j} \Lambda_{j j}=\Lambda_{i i} W_{i j}
$$

Thus, if $\Lambda_{i i} \neq \Lambda_{j j}$, then $W_{i j}=0$. Due to the block-diagonal structure of $\Lambda$ with blocks being scalar matrices $\lambda_{j}^{(i)} \operatorname{Id}_{i \times i}$ with pairwise distinct $\lambda_{j}^{(i)}$, the orthogonal matrix $W \in O(n)$ is block-diagonal with the same block structure as in $\Lambda$. The condition $W^{\top} W=$ Id implies that each $i \times i$ block is a (small) orthogonal matrix. Therefore, $W \in O_{\vec{\kappa}}=\chi_{i \geq 1} O(i)^{\kappa_{i}}$ and $U=V^{\top} W V \in V^{\top} O_{\vec{\kappa}} V$. Conversely, any $U \in V^{\top} O_{\vec{\kappa}} V$ obviously fixes $A \in \mathcal{S y m}_{n, \vec{\kappa}}$ written in the above form.

Corollary 2.2. A real symmetric matrix $A \in \mathcal{S y m}_{n}$ is in Sym ${ }_{n, \vec{\kappa}}$ if and only if its stablizer $O(n)_{A}$ under the action (2.6) is conjugate to $O_{\vec{\kappa}}=\chi_{i \geq 1} O(i)^{\kappa_{i}}$.

This characterization of $\mathcal{S y m}_{n, \vec{\kappa}}$ is used in the proof of Theorem 1.6 in Subsection 3.3.

2.4. Mean curvature of a submanifold of a Riemannian manifold. In this subsection we very briefly recall a definition of the mean curvature vector field of a submanifold of a Riemannian manifold, see [KN69, Ch. VII] for more details.

Let $(M, \boldsymbol{g})$ be a Riemannian manifold and let $X \subset M$ be its smooth $n$-dimensional submanifold. The second fundamental form of $X \subset M$ is a symmetric bilinear form $\boldsymbol{b}$ on the tangent bundle $T X$ to $X$ with values in the normal bundle $(T X)^{\perp}$ to $X$ defined at 
each point $p \in X$ by

$$
\begin{aligned}
& \boldsymbol{b}: T_{p} X \times T_{p} X \rightarrow\left(T_{p} X\right)^{\perp}, \\
& \xi, \eta \quad \mapsto\left(\nabla_{\xi} \vec{\eta}\right)^{\perp},
\end{aligned}
$$

where $\vec{\eta}$ is a smooth vector field defined on some neighborhood $U \subset M$ of $p$ and such that $\vec{\eta}_{p}=\eta, \nabla_{\xi} \vec{\eta} \in T_{p} M$ is the Levi-Civita covariant derivative of $\vec{\eta}$ along the vector $\xi \in T_{p} X$ and $\left(\nabla_{\xi} \vec{\eta}\right)^{\perp} \in\left(T_{p} X\right)^{\perp}$ is the normal component of $\nabla_{\xi} \vec{\eta} \in T_{p} M$. The result $\left(\nabla_{\xi} \vec{\eta}\right)^{\perp}$ is independent of the choice of a vector field $\vec{\eta}$ that extends $\eta \in T_{p} X$. Moreover, if $\vec{\xi}$ and $\vec{\eta}$ are smooth vector fields tangent to $X$ along some open set $U \subset X$, the dependence

$$
p \in U \mapsto \boldsymbol{b}\left(\vec{\xi}_{p}, \vec{\eta}_{p}\right) \in\left(T_{p} X\right)^{\perp}
$$

is a smooth field of normal vectors to $X$.

Let $\vec{e}=\left\{\vec{e}^{1}, \ldots, \vec{e}^{n}\right\}$ be a local frame on $X$, that is, $\vec{e}^{1}, \ldots, \vec{e}^{n}$ are smooth vector fields tangent to $X$ along some open set $U \subset X$ and such that for each $p \in U$ vectors $\vec{e}_{p}^{1}, \ldots, \vec{e}_{p}^{n} \in T_{p} X$ form a basis of the tangent space $T_{p} X$. Let $\vec{G}=\left(\boldsymbol{g}\left(\vec{e}^{i}, \vec{e}^{j}\right)\right)$ be the smooth field of matrices of the metric $\boldsymbol{g}$ written in the local frame $\vec{e}$ and let $\vec{G}^{-1}$ be the smooth field of inverses of $\vec{G}$, that is, $\vec{G}_{p}^{-1}=\left(\boldsymbol{g}\left(\vec{e}_{p}^{i}, \vec{e}_{p}^{j}\right)\right)^{-1}$ for $p \in U$. Then the mean curvature vector field of $X$ along $U$ is defined by

$$
\left.H\right|_{U}=\sum_{i, j=1}^{n}\left(\vec{G}^{-1}\right)_{i j} \boldsymbol{b}\left(\vec{e}^{i}, \vec{e}^{j}\right),
$$

where $\boldsymbol{b}\left(\vec{e}^{i}, \vec{e}^{j}\right)$ is the field of normal vectors $\boldsymbol{b}\left(\vec{e}_{p}^{i}, \vec{e}_{p}^{j}\right) \in\left(T_{p} X\right)^{\perp}, p \in U$, and the mean curvature vector of $X$ at a point $p \in U$ is given by

$$
H_{p}=\sum_{i, j=1}^{n}\left(\vec{G}_{p}^{-1}\right)_{i j} \boldsymbol{b}\left(\vec{e}_{p}^{i}, \vec{e}_{p}^{j}\right) \in\left(T_{p} X\right)^{\perp} .
$$

The definition (2.8) of $\left.H\right|_{U}$ is independent of the choice of a local frame on $U$. By gluing mean curvature vector fields (2.8) along open sets from an open cover of $X$ we obtain the smooth field $H$ of normal vectors to $X$, called the mean curvature vector field of $X$. A submanifold $X \subset M$ is called minimal if its mean curvature vector field is zero.

Remark 2.3. Usually one defines the mean curvature vector field of an $n$-dimensional submanifold $X \subset M$ as $\frac{1}{n} H$, where $H$ is defined above. However, since we are interested in minimal submanifolds, that is, when $H=0$, the factor of $\frac{1}{n}$ is unessential for us.

Example 2.4. If $X \subset\left(\mathbb{R}^{N},\langle\cdot, \cdot\rangle\right)$ is a submanifold of the Euclidean space, the covariant derivative $\nabla$ coincides with the directional derivative of components of a vector field along a vector and, in particular, the second fundamental form of $X$ computed on two vectors $\xi=\left(\xi^{1}, \ldots, \xi^{N}\right), \eta=\left(\eta^{1}, \ldots, \eta^{N}\right) \in T_{p} X \subset \mathbb{R}^{N}$ equals

$$
\boldsymbol{b}(\xi, \eta)=\left(\sum_{i=1}^{N} \xi^{i} \partial_{x_{i}} \vec{\eta}^{1}, \ldots, \sum_{i=1}^{N} \xi^{i} \partial_{x_{i}} \vec{\eta}^{N}\right)^{\perp} \in\left(T_{p} X\right)^{\perp} \subset \mathbb{R}^{N}
$$


where $\vec{\eta}=\left(\vec{\eta}^{1}, \ldots, \vec{\eta}^{N}\right)$ is a smooth vector field defined in a neighborhood of $p$ and such that $\vec{\eta}_{p}=\eta \in T_{p} X$. Consider a local parametrization of $X$, that is, a smooth map

$$
r: U \subset \mathbb{R}^{n} \rightarrow X
$$

from some open set $U \subset \mathbb{R}^{n}$ to $X$ such that the vector fields $\partial_{u_{1}} r, \ldots, \partial_{u_{n}} r$ form a local frame on $X$ along $U$. It follows from (2.9) that

$$
\boldsymbol{b}\left(\partial_{u_{i}} r, \partial_{u_{j}} r\right)=\left(\partial_{u_{i}} \partial_{u_{j}} r\right)^{\perp}, \quad i, j=1, \ldots, n \text {. }
$$

In particular, the mean curvature vector of $X$ at $r(u) \in X$ is computed as

$$
H_{r(u)}=\sum_{i, j=1}^{n}\left(\vec{G}_{r(u)}^{-1}\right)_{i j}\left(\partial_{u_{i}} \partial_{u_{j}} r(u)\right)^{\perp} \in\left(T_{r(u)} X\right)^{\perp},
$$

where $\vec{G}_{r(u)}^{-1}$ is the inverse of the matrix $\vec{G}_{r(u)}=\left(\left\langle\partial_{u_{i}} r(u), \partial_{u_{j}} r(u)\right\rangle\right)$ of the metric written in the basis $\partial_{u_{1}} r(u), \ldots, \partial_{u_{n}} r(u) \in T_{r(u)} X$. Denoting by $\mathrm{d}^{2} r(u)=\left(\partial_{u_{i}} \partial_{u_{j}} r(u)\right)$ the $n \times n$ matrix of second partial derivatives of the local parametrization (2.10), we formally write

$$
H_{r(u)}=\operatorname{Tr}\left[\vec{G}_{r(u)}^{-1}\left(\mathrm{~d}^{2} r(u)\right)^{\perp}\right],
$$

where $\left(\mathrm{d}^{2} r(u)\right)^{\perp}=\left(\left(\partial_{u_{i}} \partial_{u_{j}} r(u)\right)^{\perp}\right)$.

If $X \subset M \subset \mathbb{R}^{N}$, expressions (2.11), (2.12) and (2.13) are valid with a slight change: additionally, one needs to project vectors $\left(\partial_{u_{i}} \partial_{u_{j}} r(u)\right)^{\perp}$ to the tangent space $T_{r(u)} M$.

In our computation of mean curvature vectors of $\mathscr{M}_{m, n, r}$ and $\delta \boldsymbol{k}_{n, 2 r}$ in Subsections 3.1 and 3.2 it is more convenient to use the formal form (2.13) of the expression (2.12).

2.5. Minimality in Euclidean and projective spaces. In this subsection we give a proof of the folklore fact that a conic submanifold of an Euclidean space is minimal if and only if its intersection with a sphere is a minimal submanifold of the sphere if and only if its projectivization is a minimal submanifold of the real projective space.

Let $\left(\mathbb{R}^{N},\langle\cdot, \cdot\rangle\right)$ be an Euclidean space and denote by $S^{N-1}=\left\{p \in \mathbb{R}^{N}:\langle p, p\rangle=1\right\}$ the unit sphere in $\mathbb{R}^{N}$ endowed with the induced metric. Let $\mathbb{P}\left(\mathbb{R}^{N}\right)$ be the projective $(N-1)$-space. The standard metric on $\mathbb{P}\left(\mathbb{R}^{N}\right)$ is the push-forward metric under the double covering map $S^{N-1} \rightarrow \mathbb{P}\left(\mathbb{R}^{N}\right)$ that sends $p \in S^{N-1}$ to the line through $p$ and $-p$.

A smooth submanifold $X \subset \mathbb{R}^{N}$ is said to be conic if $t p \in P$ for any $p \in X$ and any $t \in \mathbb{R} \backslash\{0\}$. Given a conic submanifold $X \subset \mathbb{R}^{N}$, its projectivization $\mathbb{P}(X) \subset \mathbb{P}\left(\mathbb{R}^{N}\right)$ is a smooth submanifold of the projective space $\mathbb{P}\left(\mathbb{R}^{N}\right)$.

Example 2.5. The manifold $\mathscr{M}_{m, n, r}$ of $m \times n$ real matrices of rank $r$, the manifold $\delta k_{n, 2 r}$ of $n \times n$ real skew-symmetric matrices of rank $2 r$ and the manifold $\mathcal{S y m}_{n, \vec{\kappa}}$ of $n \times n$ real symmetric matrices with exactly $\kappa_{i}$ eigenvalues of multiplicity $i$, where $\vec{\kappa}=\left(\kappa_{1}, \kappa_{2}, \ldots\right)$, are conic submanifolds of $\mathscr{M}_{m, n}, \delta \varkappa_{n}$ and $\delta y m_{n}$ respectively.

The following fact is well-known, but we anyway include a proof of it.

Proposition 2.6. Let $X \subset \mathbb{R}^{N}$ be a conic submanifold. Then $X$ is minimal in $\mathbb{R}^{N}$ if and only if $X \cap S^{N-1}$ is minimal in $S^{N-1}$ if and only if $\mathbb{P}(X)$ is minimal in $\mathbb{P}\left(\mathbb{R}^{N}\right)$. 
Proof. Observe first that for any $t \in \mathbb{R} \backslash\{0\}$ the dilation

$$
\begin{aligned}
\delta_{t}: \mathbb{R}^{N} & \rightarrow \mathbb{R}^{N} \\
p & \mapsto t p
\end{aligned}
$$

is a homothety of $\left(\mathbb{R}^{N},\langle\cdot, \cdot\rangle\right)$. Thus to prove that a conic submanifold $X \subset \mathbb{R}^{N}$ is minimal it is enough to show that the mean curvature vector $H_{p}$ of $X$ is zero at any $p \in X \cap S^{N-1}$. Now, the tangent space to the sphere at $p \in S^{N-1}$ is identified with the space of vectors orthogonal to $p$, that is, $T_{p} S^{N-1}=p^{\perp}=\left\{\xi \in \mathbb{R}^{N}:\langle\xi, p\rangle=0\right\}$. Under this identification and in view of the fact that $p \in T_{p} X$, at any $p \in X \cap S^{N-1}$ the normal spaces to $X \subset \mathbb{R}^{N}$ and to $X \cap S^{N-1} \subset S^{N-1}$ coincide, $\left(T_{p} X\right)^{\perp}=\left(T_{p}\left(X \cap S^{N-1}\right)\right)^{\perp} \subset p^{\perp} \subset \mathbb{R}^{N}$.

Assume that $X$ has dimension $n$ and consider a local parametrization $r: U \rightarrow X \cap S^{N-1}$ of $X \cap S^{N-1}$ near $p$ such that $0 \in U \subset \mathbb{R}^{n-1}$ and $r(0)=p$. Then the map

$$
\begin{aligned}
R: U \times(-\varepsilon, \varepsilon) & \rightarrow X, \\
u=\left(u_{1}, \ldots, u_{n-1}\right), u_{n} & \mapsto\left(1+u_{n}\right) r(u),
\end{aligned}
$$

is a local parametrization of $X$ near $p$ such that $R(0)=p$. The metric $G=\left(\left\langle\partial_{u_{i}} R(0), \partial_{u_{j}} R(0)\right\rangle\right)$ written in the basis

$$
\partial_{u_{1}} R(0)=\partial_{u_{1}} r(0), \ldots, \partial_{u_{n-1}} R(0)=\partial_{u_{n-1}} r(0), \partial_{u_{n}} R(0)=p
$$

has a block-diagonal form

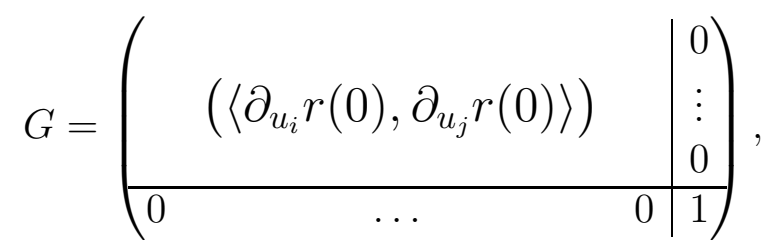

where the upper-left block $g=\left(\left\langle\partial_{u_{i}} r(0), \partial_{u_{j}} r(0)\right\rangle\right)$ is the metric on $T_{p}\left(X \cap S^{N-1}\right)$.

Since $\partial_{u_{n}} \partial_{u_{n}} R(0)=0$ and $\partial_{u_{i}} \partial_{u_{n}} R(0)=\partial_{u_{i}} r(0) \in T_{p}\left(X \cap S^{N-1}\right), i=1, \ldots, n-1$, formula (2.11) implies that the matrix of the second fundamental form of $X \subset \mathbb{R}^{N}$ at $p$ computed in the basis (2.14) has the form

$$
\left(\mathrm{d}^{2} R(0)\right)^{\perp}=\left(\begin{array}{cc|c}
\left(\left(\partial_{u_{i}} \partial_{u_{j}} r(0)\right)^{\perp}\right) & & \vdots \\
& & 0 \\
\hline 0 & 0 & 0
\end{array}\right) .
$$

Since the normal spaces to $X$ and $X \cap S^{N-1}$ at $p$ coincide, $\left(\partial_{u_{i}} \partial_{u_{j}} r(0)\right)^{\perp} \in\left(T_{p} X\right)^{\perp}=$ $\left(T_{p}\left(X \cap S^{N-1}\right)\right)^{\perp}$ is the value of the second fundamental form of both $X \subset \mathbb{R}^{N}$ and $X \cap S^{N-1} \subset S^{N-1}$. Thus the upper-left block in (2.15) is the matrix $\left(\mathrm{d}^{2} r(0)\right)^{\perp}=$ $\left(\left(\partial_{u_{i}} \partial_{u_{j}} r(0)\right)^{\perp}\right)$ of the second fundamental form of $X \cap S^{N-1} \subset S^{N-1}$ at $p$ written in the basis $\partial_{u_{1}} r(0), \ldots, \partial_{u_{n-1}} r(0)$ of $T_{p}\left(X \cap S^{N-1}\right)$. As a consequence, the mean curvature 
vectors of $X \subset \mathbb{R}^{N}$ and $X \cap S^{N-1} \subset S^{N-1}$ at $p$ are equal,

$$
H(X)_{p}=\operatorname{Tr}\left(G^{-1}\left(\mathrm{~d}^{2} R(0)\right)^{\perp}\right)=\operatorname{Tr}\left(g^{-1}\left(\mathrm{~d}^{2} r(0)\right)^{\perp}\right)=H\left(X \cap S^{N-1}\right)_{p} .
$$

In particular, a conic submanifold $X \subset \mathbb{R}^{N}$ is minimal if and only if its intersection with the sphere $X \cap S^{N-1}$ is minimal in $S^{N-1}$. Finally, since the double covering $S^{N-1} \rightarrow \mathbb{P}\left(\mathbb{R}^{N}\right)$ is, by construction, a local isometry, and since the definition of the mean curvature is also local, the second equivalence in the statement of the proposition follows.

In Section 1 we use Proposition 2.6 to derive Corollaries 1.2, 1.4 and 1.7 from Theorems $1.1,1.3$ and 1.6 respectively.

\section{Proof of MAin Results}

In this section we prove Theorems 1.1, 1.3 and 1.6. Proofs of Theorems 1.1 and 1.3 are performed in local coordinates given by normal forms (2.2) and (2.4), while the proof of Theorem 1.6 is derived from a general result of Hsiang and Lawson from [HL71].

3.1. Proof of Theorem 1.1. We write a matrix $A \in \mathscr{M}_{m, n, r}$ in the SVD form (2.2) $A=U \Sigma V^{\top}$, where $U \in O(m), V \in O(n)$ and

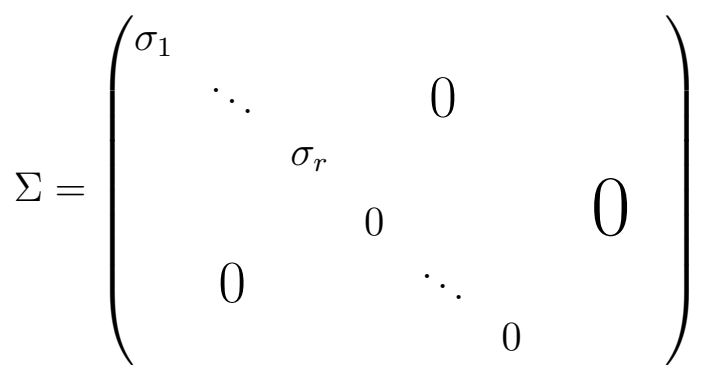

is an $m \times n$ diagonal matrix of singular values of $A$. We first assume that the nonzero singular values are ordered and distinct, that is, $\sigma_{1}>\cdots>\sigma_{r}>0$. Recall from Section 2 that the inner product (1.1) is invariant under the action (2.1) of $O(m) \times O(n)$. Therefore, to prove that $\mathscr{M}_{m, n, r}$ is a minimal submanifold of $\left(\mathscr{M}_{m, n},\langle\cdot, \cdot\rangle\right)$ it is enough to show that for any diagonal matrix $A=\Sigma \in \mathscr{M}_{m, n, r}$ the mean curvature vector (2.13) at $A=\Sigma$ is zero. For this we consider the following parametrization of a neighborhood of $A=\Sigma$ :

$$
A(\boldsymbol{\mu}, \boldsymbol{s}, \boldsymbol{\nu})=\left(\prod_{\substack{1 \leq i<j \leq m, i \leq r}} e^{\mu_{i j} L_{i j}}\right)\left(\Sigma+\sum_{h=1}^{r} s_{h} E_{h h}\right)\left(\prod_{\substack{1 \leq k<\ell \leq n, k \leq r}} e^{-\nu_{k \ell} L_{k \ell}}\right),
$$

where $E_{i j}$ denotes the $(i, j)$ th matrix unit, $L_{i j}=E_{j i}-E_{i j}$ and matrices $e^{\mu_{i j} L_{i j}}$ and $e^{-\nu_{k \ell} L_{k \ell}}$ in the two products are ordered according to the lexicographic order on sets of indices $(i, j)$ and $(k, \ell)$. Note that $\mu \mapsto e^{\mu L_{i j}}$ is a smooth one-parameter subgroup of orthogonal matrices such that $e^{0 L_{i j}}=\mathrm{id}$ and $\frac{d}{d \mu} e^{\mu L_{i j}}=L_{i j} e^{\mu L_{i j}}=e^{\mu L_{i j}} L_{i j}$. In particular, 
$A(\mathbf{0}, \mathbf{0}, \mathbf{0})=A=\Sigma$ and $\left.\frac{d}{d \mu}\right|_{\mu=0} e^{\mu L_{i j}}=L_{i j}$. Using this we now compute first order derivatives of the parametrization (3.1). We have

$$
\partial_{\mu_{i j}} A(\boldsymbol{\mu}, \boldsymbol{s}, \boldsymbol{\nu})=\left(e^{\mu_{12} L_{12}} \cdots e^{\mu_{i j} L_{i j}} L_{i j} \cdots e^{\mu_{r m} L_{r m}}\right)\left(\Sigma+\sum_{h=1}^{r} s_{h} E_{h h}\right)\left(\prod_{\substack{1 \leq k<\ell \leq n, k \leq r}} e^{-\nu_{k \ell} L_{k \ell}}\right)
$$

for $1 \leq i<j \leq m$ with $i \leq r$,

$$
\partial_{s_{h}} A(\boldsymbol{\mu}, \boldsymbol{s}, \boldsymbol{\nu})=\left(\prod_{\substack{1 \leq i<j \leq m, i \leq r}} e^{\mu_{i j} L_{i j}}\right) E_{h h}\left(\prod_{\substack{1 \leq k<\ell \leq n, k \leq r}} e^{-\nu_{k \ell} L_{k \ell}}\right)
$$

for $1 \leq h \leq r$ and

$$
\partial_{\nu_{k \ell}} A(\boldsymbol{\mu}, \boldsymbol{s}, \boldsymbol{\nu})=\left(\prod_{\substack{1 \leq i<j \leq m, i \leq r}} e^{\mu_{i j} L_{i j}}\right)\left(\Sigma+\sum_{h=1}^{r} s_{h} E_{h h}\right)\left(e^{-\nu_{12} L_{12}} \cdots\left(-L_{k \ell}\right) e^{-\mu_{k \ell} L_{k \ell}} \cdots e^{-\mu_{r n} L_{r n}}\right)
$$

for $1 \leq k<\ell \leq n$ with $k \leq r$. Note that matrices (3.2), (3.3) and (3.4) belong to the tangent space to $\mathscr{M}_{m, n, r}$ at the point $A(\boldsymbol{\mu}, \boldsymbol{s}, \boldsymbol{\nu})$. At $A(\mathbf{0}):=A(\mathbf{0}, \mathbf{0}, \mathbf{0})=A=\Sigma$ these are equal

$$
\begin{aligned}
\partial_{\mu_{i j}} A(\mathbf{0})=L_{i j} \Sigma=\sigma_{i} E_{j i}-\sigma_{j} E_{i j}, & 1 \leq i<j \leq m, i \leq r, \\
\partial_{s_{h}} A(\mathbf{0})=E_{h h}, & 1 \leq h \leq r, \\
\partial_{\nu_{k \ell}} A(\mathbf{0})=\Sigma\left(-L_{k \ell}\right)=\sigma_{k} E_{k \ell}-\sigma_{\ell} E_{\ell k}, & 1 \leq k<\ell \leq n, k \leq r,
\end{aligned}
$$

where we set $\sigma_{j}=\sigma_{\ell}=0$ for $j, \ell>r$. There are

$$
\left(\begin{array}{c}
m \\
2
\end{array}\right)-\left(\begin{array}{c}
m-r \\
2
\end{array}\right)+r+\left(\begin{array}{l}
n \\
2
\end{array}\right)-\left(\begin{array}{c}
n-r \\
2
\end{array}\right)=(m+n) r-r^{2}
$$

matrices in (3.5) and it is easy to see that they are linearly independent. The count (3.6) and the formula $\operatorname{dim}\left(\mathscr{M}_{m, n, r}\right)=(m+n) r-r^{2}$ [Har92] imply that (3.1) is indeed a parametrization of $\mathscr{M}_{m, n, r}$ around $A(\mathbf{0})=A$ and, in particular, matrices (3.5) form a basis of the tangent space to $\mathscr{M}_{m, n, r}$ at $A=\Sigma$. We now compute the metric tensor of $\mathscr{M}_{m, n, r}$ at $A$ in this basis. Let us observe that matrices $\partial_{\mu_{i j}} A(\mathbf{0})$ are orthogonal among themselves. The same holds for $\partial_{s_{h}} A(\mathbf{0})$ and for $\partial_{\nu_{k \ell}} A(\mathbf{0})$. Furthermore, matrices $\partial_{\mu_{i j}} A(\mathbf{0})$ any $\partial_{\nu_{k \ell}} A(\mathbf{0})$ are orthogonal to $\partial_{s_{h}} A(\mathbf{0})$. Finally, $\partial_{\mu_{i j}} A(\mathbf{0})$ is orthogonal to $\partial_{\nu_{k \ell}} A(\mathbf{0})$ unless $i=k$ and $j=\ell \leq r$ in which case their inner product equals $\left\langle\partial_{\mu_{i j}} A(\mathbf{0}), \partial_{\nu_{i j}} A(\mathbf{0})\right\rangle=-2 \sigma_{i} \sigma_{j}$. Summarizing, in the basis (3.5) the metric tensor $G$ has the following block-diagonal form 


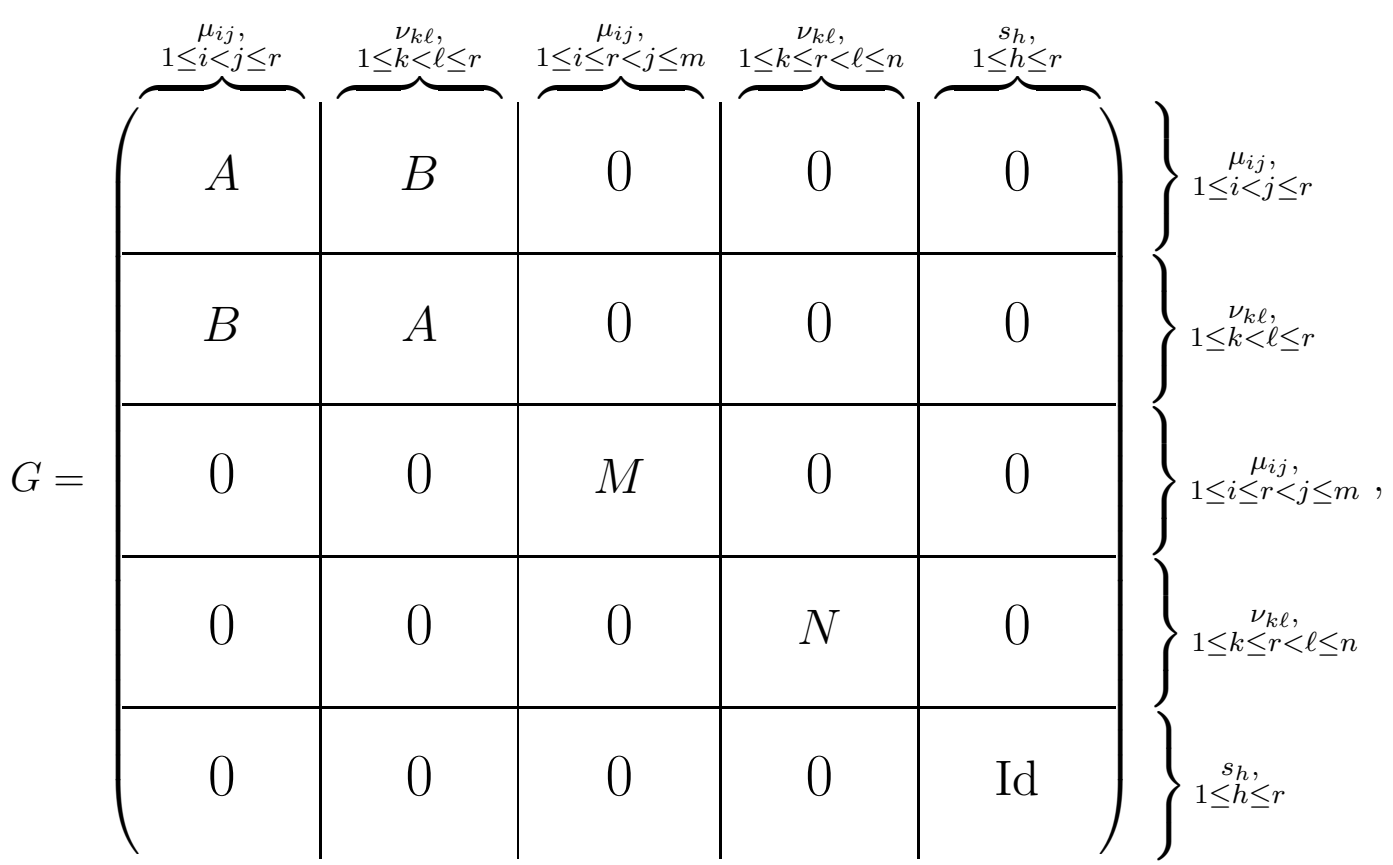

where the order in each of the indicated groups of rows and columns of $G$ is induced from the lexicographic order on sets of indices $(i, j),(k, \ell)$ and

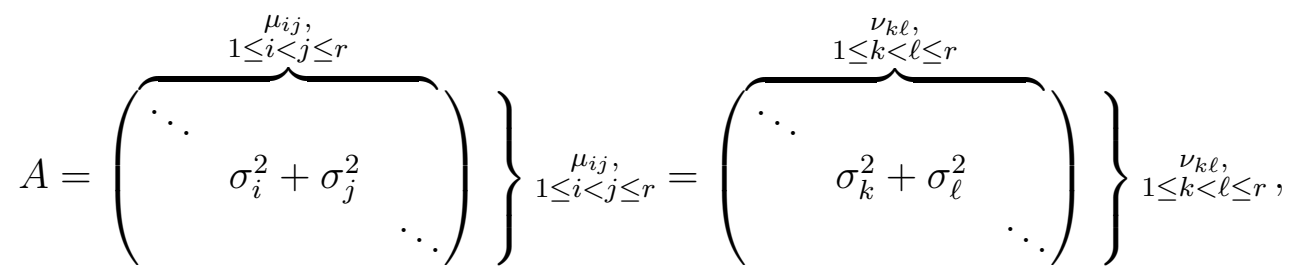

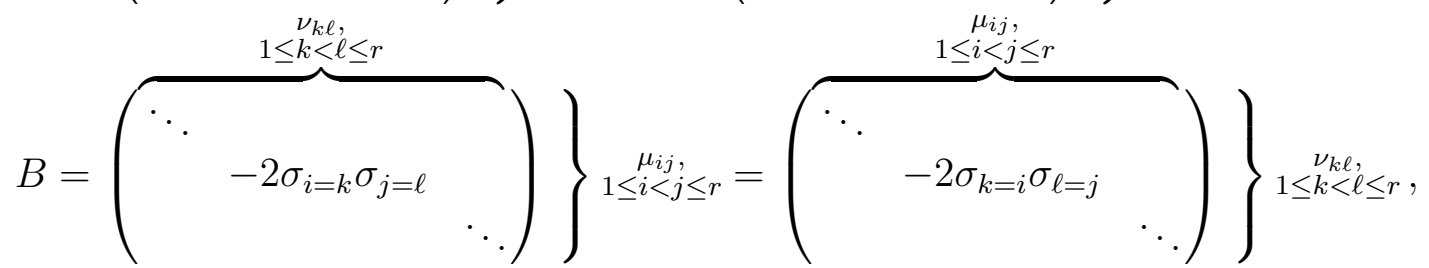

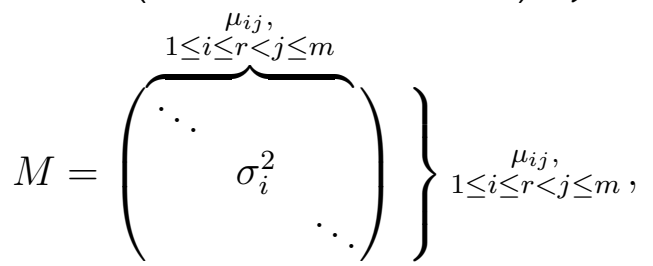

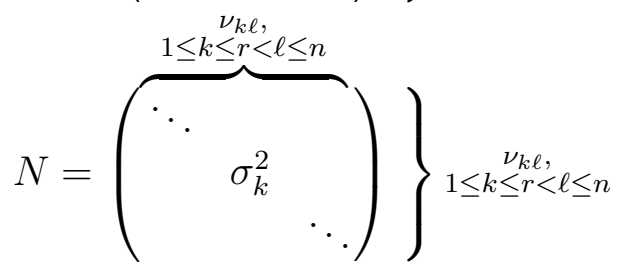

are diagonal square matrices of sizes $\left(\begin{array}{c}r \\ 2\end{array}\right),\left(\begin{array}{c}r \\ 2\end{array}\right), r(m-r), r(n-r)$ respectively. Since $G$ is block-diagonal and blocks $A, B, M$ and $N$ are diagonal matrices, the inverse of $G$ equals 
ON MINIMALITY OF DETERMINANTAL VARIETIES

\begin{tabular}{|c|c|c|c|c|c|c|}
\hline \multirow[t]{3}{*}{$(3.8)$} & $\begin{array}{c}\mu_{i j}, \\
1 \leq i<j \leq r\end{array}$ & $\begin{array}{c}\nu_{k \ell \ell}, \\
1 \leq k<\ell \leq r\end{array}$ & $\begin{array}{c}\mu_{i j}, \\
1 \leq i \leq r<j \leq m\end{array}$ & 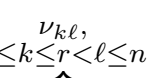 & $1 \leq h \leq r$ & \\
\hline & $\frac{A}{A^{2}-B^{2}}$ & $-\frac{B}{A^{2}-B^{2}}$ & 0 & 0 & 0 & $\begin{array}{c}\mu_{i j} \\
1 \leq i<j \leq r\end{array}$ \\
\hline & $-\frac{B}{A^{2}-B^{2}}$ & $\frac{A}{A^{2}-B^{2}}$ & 0 & 0 & 0 & $\begin{array}{c}\nu_{k \ell} \\
1 \leq k<\ell \leq r\end{array}$ \\
\hline \multirow[t]{3}{*}{$G^{-1}=$} & 0 & 0 & $M^{-1}$ & 0 & 0 & $\begin{array}{c}\mu_{i j}, \\
1 \leq i \leq r<j \leq m\end{array}$ \\
\hline & 0 & 0 & 0 & $N^{-1}$ & 0 & $\begin{array}{c}\nu_{k \ell}, \\
1 \leq k \leq r<\ell \leq n\end{array}$ \\
\hline & 0 & 0 & 0 & 0 & Id & $\begin{array}{c}s_{h} \\
1 \leq h \leq r\end{array}$ \\
\hline
\end{tabular}

with diagonal blocks

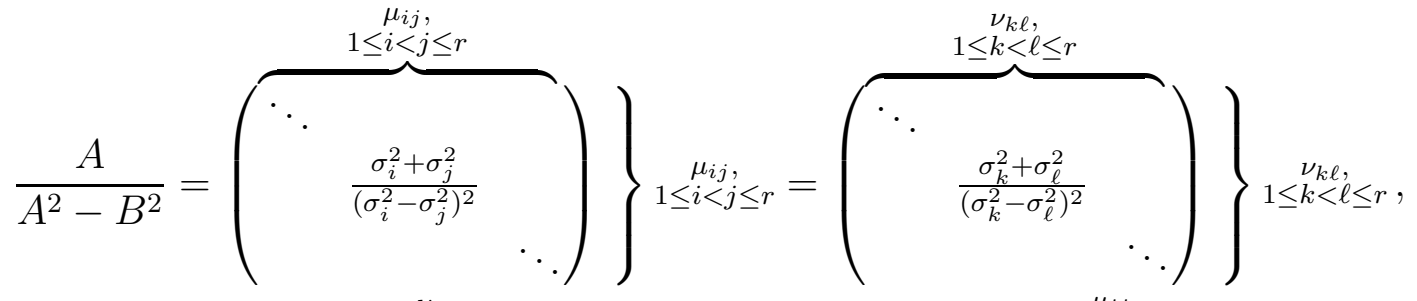

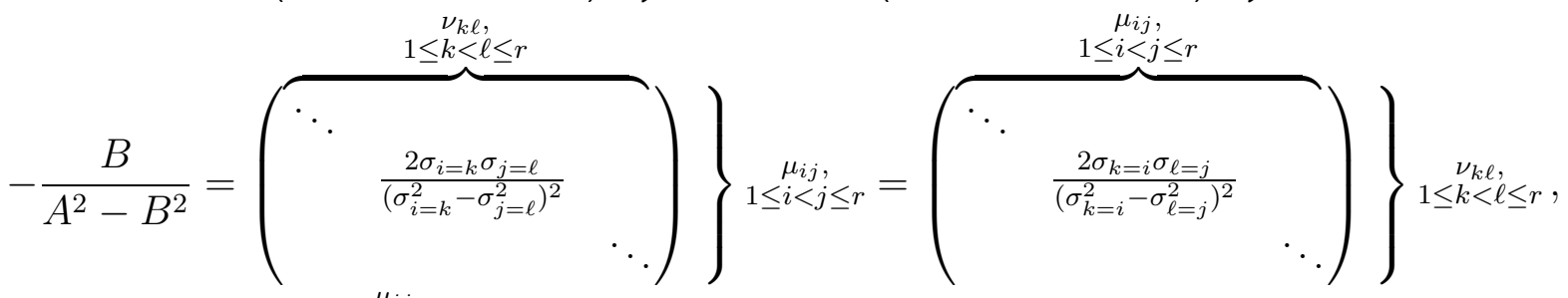

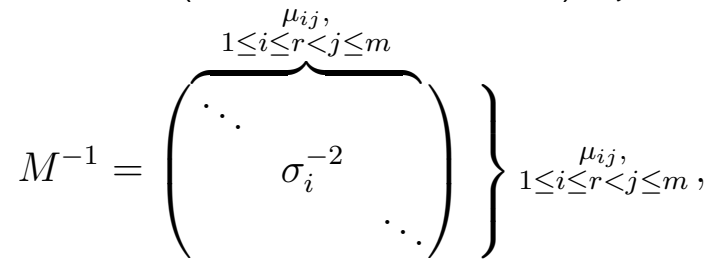

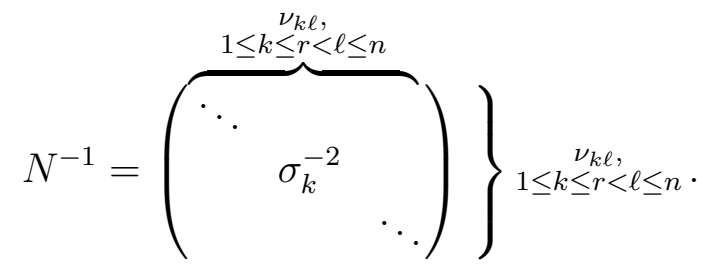


Now, (2.13) implies that the mean curvature vector of $\mathscr{M}_{m, n, r}$ at $A$ is equal to

$$
H_{A}=\operatorname{Tr}\left[G^{-1}\left(\mathrm{~d}^{2} A(\mathbf{0})\right)^{\perp}\right]
$$

where $\mathrm{d}^{2} A(\mathbf{0})$ is the matrix of second partial derivatives of (3.1) computed at $(\mu, \nu, s)=$ $(\mathbf{0}, \mathbf{0}, \mathbf{0})$ and $\left(\mathrm{d}^{2} A(\mathbf{0})\right)^{\perp}$ stands for its normal component (applied entry-wise to $\mathrm{d}^{2} A(\mathbf{0})$ ). From (2.12) we see that in order to compute $H_{A}$ we need only those second partial derivatives of (3.1) that correspond to non-zero entries of $G^{-1}$. For $1 \leq i<j \leq m$, $i \leq r$, for $1 \leq h \leq r$ and for $1 \leq k<\ell \leq n, k \leq r$, we have, using (3.2), (3.3) and (3.4),

$$
\begin{aligned}
\partial_{\mu_{i j}} \partial_{\mu_{i j}} A(\mathbf{0}) & =L_{i j}^{2}\left(\sum_{h=1}^{r} \sigma_{h} E_{h h}\right)=\left(-E_{i i}-E_{j j}\right)\left(\sum_{h=1}^{r} \sigma_{h} E_{h h}\right)=-\sigma_{i} E_{i i}-\sigma_{j} E_{j j}, \\
\partial_{s_{h}} \partial_{s_{h}} A(\mathbf{0}) & =0 \\
\partial_{\nu_{k \ell}} \partial_{\nu_{k \ell}} A(\mathbf{0}) & =\left(\sum_{h=1}^{r} \sigma_{h} E_{h h}\right) L_{k \ell}^{2}=\left(\sum_{h=1}^{r} \sigma_{h} E_{h h}\right)\left(-E_{k k}-E_{\ell \ell}\right)=-\sigma_{k} E_{k k}-\sigma_{\ell} E_{\ell \ell},
\end{aligned}
$$

where we recall that $\sigma_{j}=\sigma_{\ell}=0$ for $j, \ell>r$. Finally, for $1 \leq i=k<j=\ell \leq r$ we have

$$
\partial_{\mu_{i j}} \partial_{\nu_{i j}} A(\mathbf{0})=L_{i j}\left(\sum_{h=1}^{r} \sigma_{h} E_{h h}\right)\left(-L_{i j}\right)=\sigma_{j} E_{i i}+\sigma_{i} E_{j j}
$$

From the form (3.8) of $G^{-1}$ we see that (3.10) and (3.11) are the only second partial derivatives of (3.1) that matter for the formula (3.9) of $H_{A}$. Before we proceed to computing normal components of these matrices let us describe the normal space to $\mathscr{M}_{m, n, r}$ at $A=A(\mathbf{0})$. Matrices

$$
E_{p q}, \quad r<p \leq m, r<q \leq n,
$$

are orthogonal to matrices (3.5) that form a basis of the tangent space $T_{A} \mathscr{M}_{m, n, r}$. Moreover, (3.12) are independent and there are $(m-r)(n-r)=m n-\left((m+n) r-r^{2}\right)=\operatorname{codim}\left(\mathscr{M}_{m, n, r}\right)$ many of them. It implies that matrices (3.12) form a basis of the normal space to $\mathscr{M}_{m, n, r}$ at $A$. It is now straightforward to see that (3.10) and (3.11) are orthogonal to (3.12) or, equivalently, they have trivial normal components. It follows then from the above reasoning that $H_{A}=0$.

In the beginning of the proof we assumed that the non-zero singular values of $A \in \mathscr{M}_{m, n, r}$ are distinct. Those $A \in \mathscr{M}_{m, n, r}$ that do not satisfy this assumption form an algebraic submanifold $X \subset \mathscr{M}_{m, n, r}$ which is proper because there are obviously matrices in $\mathscr{M}_{m, n, r} \backslash X$. Since the mean curvature vector field $H$ is a smooth field of normal vectors to $\mathscr{M}_{m, n, r}$ and since $H_{A}=0$ for $A$ in the open and dense subset $\mathscr{M}_{m, n, r} \backslash X \subset \mathscr{M}_{m, n, r}$, we have $H_{A}=0$ for all $A \in \mathscr{M}_{m, n, r}$.

3.2. Proof of Theorem 1.3. We pursue the same strategy as in the proof of Theorem 1.1 . Let us write a skew-symmetric matrix $A \in \delta \xi_{n, r}$ of rank $2 r<n$ in the normal form (2.4), $A=V^{\top} \Omega V$, where $V \in O(n)$ and

$$
\Omega=\sum_{h=1}^{r} \omega_{h} L_{2 h, 2 h-1}
$$

is as in (2.5), where recall $L_{i j}=E_{j i}-E_{i j}$. We first assume that $\omega_{1}>\cdots>\omega_{r}>0$. Since the Frobenius inner product (1.1) is invariant under the action (2.1) of $O(n) \times O(n)$ on $\mathscr{M}_{n, n}$ it is, in particular, invariant under the diagonal subaction (2.3) of $O(n)$ on $\delta k_{n}$. Thus, in order to prove that $\delta k_{n, 2 r}$ is a minimal submanifold of $\left(\delta k_{n},\langle\cdot, \cdot\rangle\right)$ it is enough to show that for any 
block-diagonal matrix $A=\Omega \in \delta k_{n, 2 r}(3.13)$ the mean curvature vector (2.13) at $A=\Omega$ is zero. For this let us consider the following parametrization of a neighborhood of $A=\Omega$ :

$$
A(\boldsymbol{\mu}, \boldsymbol{s})=\left(\prod_{\substack{1 \leq i<j \leq n, i \leq 2 r,(i, j) \neq(2 t-1,2 t)}} e^{\mu_{i j} L_{i j}}\right)^{\top}\left(\sum_{h=1}^{r}\left(\omega_{h}+s_{h}\right) L_{2 h, 2 h-1}\right)\left(\prod_{\substack{1 \leq k<\ell \leq n, k \leq 2 r,(k, \ell) \neq(2 t-1,2 t)}} e^{\mu_{k \ell} L_{k \ell}}\right),
$$

where orthogonal matrices $e^{\mu_{i j} L_{i j}}$ in the product in (3.14) are ordered according to the lexicographic order on the set of indices $(i, j)$ and $(i, j) \neq(2 t-1,2 t)$ means that $i<j$ are not consecutive integers with $i$ being odd. Exactly in the same way as in the proof of Theorem 1.1 we compute first order derivatives of the parametrization (3.14). We have

$$
\begin{aligned}
\partial_{\mu_{i j}} A(\boldsymbol{\mu}, \boldsymbol{s}) & =\left(e^{\mu_{13} L_{13}} \ldots L_{i j} e^{\mu_{i j} L_{i j}} \ldots e^{\mu_{2 r, n} L_{2 r, n}}\right)^{\top}\left(\sum_{h=1}^{r}\left(\omega_{h}+s_{h}\right) L_{2 h, 2 h-1}\right)\left(\prod_{\substack{1 \leq k<\ell \leq n, k \leq 2 r,(k, \ell) \neq(2 t-1,2 t)}} e^{\mu_{k \ell} L_{k \ell}}\right) \\
& +\left(\prod_{\substack{1 \leq k<\ell \leq n, k \leq 2 r,(k, \ell) \neq(2 t-1,2 t)}} e^{\mu_{k \ell} L_{k \ell}}\right)^{\top}\left(\sum_{h=1}^{r}\left(\omega_{h}+s_{h}\right) L_{2 h, 2 h-1}\right)\left(e^{\mu_{13} L_{13}} \ldots L_{i j} e^{\mu_{i j} L_{i j}} \ldots e^{\mu_{2 r, n} L_{2 r, n}}\right)
\end{aligned}
$$

for $1 \leq i<j \leq n, i \leq 2 r$ with $(i, j) \neq(2 t-1,2 t)$ and

$$
\partial_{s_{h}} A(\boldsymbol{\mu}, \boldsymbol{s})=\left(\prod_{\substack{1 \leq i<j \leq n, i \leq 2 r,(i, j) \neq(2 t-1,2 t)}} e^{\mu_{i j} L_{i j}}\right)^{\top} L_{2 h, 2 h-1}\left(\prod_{\substack{1 \leq k<\ell \leq n, k \leq 2 r,(k, \ell) \neq(2 t-1,2 t)}} e^{\mu_{k \ell} L_{k \ell}}\right)
$$

for $1 \leq h \leq r$. Matrices (3.15), (3.16) belong to the tangent space to $\delta k_{n, 2 r}$ at $A(\boldsymbol{\mu}, \boldsymbol{s})$. At $A(\mathbf{0}):=A(\mathbf{0}, \mathbf{0})=A=\Omega$ these are equal

$$
\begin{aligned}
\partial_{\mu_{i j}} A(\mathbf{0}) & =-L_{i j} \Omega+\Omega L_{i j}=\sum_{h=1}^{r} \omega_{h}\left(L_{2 h, 2 h-1} L_{i j}-L_{i j} L_{2 h, 2 h-1}\right) \\
& =\sum_{h=1}^{r} \omega_{h}\left(L_{i, 2 h-1} \delta_{j, 2 h}-L_{j, 2 h-1} \delta_{i, 2 h}-L_{i, 2 h} \delta_{j, 2 h-1}+L_{j, 2 h} \delta_{i, 2 h-1}\right)
\end{aligned}
$$

for $1 \leq i<j \leq n, i \leq 2 r,(i, j) \neq(2 t-1,2 t)$, where $\delta_{i j}$ is the Kronecker delta symbol, and

$$
\partial_{s_{h}} A(\mathbf{0})=L_{2 h, 2 h-1}, \quad 1 \leq h \leq r .
$$

We now elaborate (3.17) further and distinguish the following four cases depending on the parity of the indices $i$ and $j$ :

(i) $1 \leq i=2 p<j=2 q \leq n, p \leq r$, (both $i$ and $j$ are even)

$$
\partial_{\mu_{i j}} A(\mathbf{0})=\omega_{p} L_{2 p-1,2 q}+\omega_{q} L_{2 p, 2 q-1}
$$

(ii) $1 \leq i=2 p<j=2 q-1 \leq n, p \leq r,(i$ is even and $j$ is odd $)$

$$
\partial_{\mu_{i j}} A(\mathbf{0})=\omega_{p} L_{2 p-1,2 q-1}-\omega_{q} L_{2 p, 2 q}
$$


(iii) $1 \leq i=2 p-1<j=2 q \leq n, p \leq r, p<q$, ( $i$ is odd and $j$ is even $)$

$$
\partial_{\mu_{i j}} A(\mathbf{0})=-\omega_{p} L_{2 p, 2 q}+\omega_{q} L_{2 p-1,2 q-1}
$$

(iv) $1 \leq i=2 p-1<j=2 q-1 \leq n, p \leq r$, (both $i$ and $j$ are odd)

$$
\partial_{\mu_{i j}} A(\mathbf{0})=-\omega_{p} L_{2 p, 2 q-1}-\omega_{q} L_{2 p-1,2 q}
$$

where we set $\omega_{q}=0$ whenever $r<q$. If $2 r<j$ (equivalently, $r<q$ ), then one can write

$$
\begin{aligned}
& \partial_{\mu_{i j}} A(\mathbf{0})=\omega_{\left\lfloor\frac{i+1}{2}\right\rfloor} L_{i-1, j} \quad \text { for even } i \text { and } \\
& \partial_{\mu_{i j}} A(\mathbf{0})=-\omega_{\left\lfloor\frac{i+1}{2}\right\rfloor} L_{i+1, j} \quad \text { for odd } i .
\end{aligned}
$$

There are

$$
\left(\begin{array}{l}
n \\
2
\end{array}\right)-\left(\begin{array}{c}
n-2 r \\
2
\end{array}\right)-r+r=\left(\begin{array}{l}
n \\
2
\end{array}\right)-\left(\begin{array}{c}
n-2 r \\
2
\end{array}\right)
$$

matrices in (3.17) and (3.18) and one can see that they are linearly independent using (3.19), (3.20), (3.21), (3.22). The count (3.24) and the formula $\operatorname{dim}\left(\delta k_{n, 2 r}\right)=\left(\begin{array}{c}n \\ 2\end{array}\right)-\left(\begin{array}{c}n-2 r \\ 2\end{array}\right)$ [FL83, p.49] imply that (3.14) is indeed a parametrization of $\delta k_{n, 2 r}$ around $A(\mathbf{0})=A$ and, in particular, matrices (3.17) and (3.18) form a basis of the tangent space to $\delta k_{n, 2 r}$ at $A=\Omega$. We want to compute the metric tensor of $\delta k_{n, 2 r}$ at $A$ in this basis. For this let us first fix a particular order of the tangent vectors (3.17) and (3.18). For each pair of indices $(p, q)$ such that $1 \leq p<q \leq r$ we form the group $\left\{\partial_{\mu_{2 p-1,2 q-1}} A(\mathbf{0}), \partial_{\mu_{2 p-1,2 q}} A(\mathbf{0}), \partial_{\mu_{2 p, 2 q-1}} A(\mathbf{0}), \partial_{\mu_{2 p, 2 q}} A(\mathbf{0})\right\}$ of four tangent vectors. We then order these $(p, q)$-groups according to the lexicographic order on the set of indices $(p, q), 1 \leq p<q \leq r$. Next, we put lexicographically ordered vectors $\partial_{\mu_{i j}} A(\mathbf{0})$, $1 \leq i \leq 2 r<j \leq n$. Finally, we put $\partial_{s_{h}} A(\mathbf{0}), 1 \leq h \leq r$. From (3.17) and (3.18) we see that vectors $\partial_{s_{h}} A(\mathbf{0})$ are orthogonal to all $\partial_{\mu_{i j}} A(\mathbf{0})$ and they are also orthogonal among themselves with $\left\langle\partial_{s_{h}} A(\mathbf{0}), \partial_{s_{h}} A(\mathbf{0})\right\rangle=2$. Each $\partial_{\mu_{i j}} A(\mathbf{0}), 1 \leq i \leq 2 r<j \leq n$, is orthogonal to all other vectors and $\left\langle\partial_{\mu_{i j}} A(\mathbf{0}), \partial_{\mu_{i j}} A(\mathbf{0})\right\rangle=2 \omega_{\left\lfloor\frac{i+1}{2}\right\rfloor}^{2}$. Vectors from two different $(p, q)$-groups are orthogonal to each other and the Gram matrix of a given $(p, q)$-group equals

$$
\left.2 G_{p, q}=2\left(\begin{array}{cccc}
\overbrace{\omega_{p}^{2}+\omega_{q}^{2}}^{(2 p-1,2 q-1)} & \overbrace{0}^{(2 p-1,2 q)} & \overbrace{0}^{(2 p, 2 q-1)} & \overbrace{-2 \omega_{p} \omega_{q}}^{(2 p, 2 q)} \\
0 & \omega_{p}^{2}+\omega_{q}^{2} & 2 \omega_{p} \omega_{q} & 0 \\
0 & 2 \omega_{p} \omega_{q} & \omega_{p}^{2}+\omega_{q}^{2} & 0 \\
-2 \omega_{p} \omega_{q} & 0 & 0 & \omega_{p}^{2}+\omega_{q}^{2}
\end{array}\right)\right\}\left\{\begin{array}{l}
(2 p-1,2 q-1) \\
(2 p-1,2 q) \\
(2 p, 2 q-1) \\
(2 p, 2 q)
\end{array} .\right.
$$

Summarizing, the metric tensor $G$ in the basis (3.17), (3.18) equals 


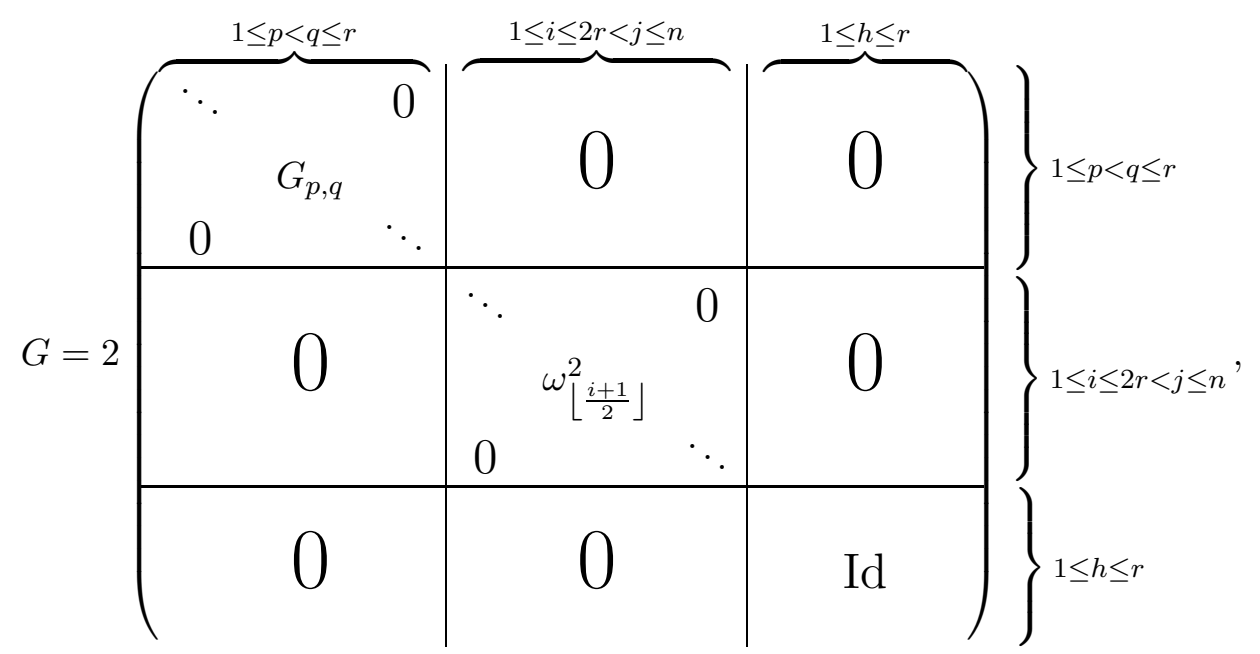

where the upper-left corner is block-diagonal with $4 \times 4$ blocks $G_{p, q}$ from (3.25). Since $G$ has block-diagonal form its inverse $G^{-1}$ equals

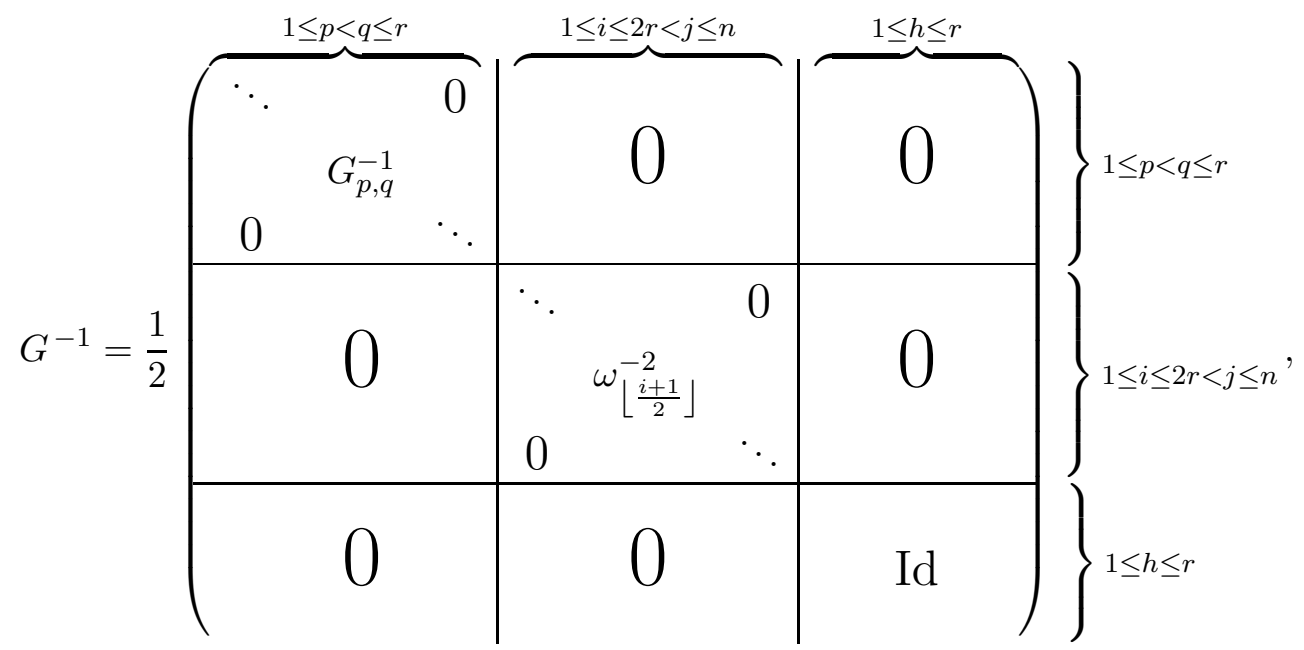

where inverses of blocks $G_{p, q}$ are easily found to be

$$
\left.G_{p, q}^{-1}=\frac{1}{\left(\omega_{p}^{2}-\omega_{q}^{2}\right)^{2}}\left(\begin{array}{cccc}
\overbrace{\omega_{p}^{2}+\omega_{q}^{2}}^{(2 p-1,2 q-1)} & \overbrace{0}^{(2 p-1,2 q)} & \overbrace{0}^{(2 p, 2 q-1)} & \overbrace{2 \omega_{p} \omega_{q}}^{(2 p, 2 q)} \\
0 & \omega_{p}^{2}+\omega_{q}^{2} & -2 \omega_{p} \omega_{q} & 0 \\
0 & -2 \omega_{p} \omega_{q} & \omega_{p}^{2}+\omega_{q}^{2} & 0 \\
2 \omega_{p} \omega_{q} & 0 & 0 & \omega_{p}^{2}+\omega_{q}^{2}
\end{array}\right)\right\}\left\{\begin{array}{l}
(2 p-1,2 q-1) \\
(2 p-1,2 q) \\
(2 p, 2 q-1) \\
(2 p, 2 q)
\end{array}\right.
$$

Recall from (2.13) that the mean curvature of $\delta k_{n, 2 r}$ at $A$ can be computed as

$$
H_{A}=\operatorname{Tr}\left[G^{-1}\left(\mathrm{~d}^{2} A(\mathbf{0})\right)^{\perp}\right]
$$

where $\mathrm{d}^{2} A(\mathbf{0})$ is the matrix of second partial derivatives of 3.14 computed at $(\mu, s)=(\mathbf{0}, \mathbf{0})$ and $\left(\mathrm{d}^{2} A(\mathbf{0})\right)^{\perp}$ stands for its normal component (applied entry-wise to $\left.\mathrm{d}^{2} A(\mathbf{0})\right)$. As in the proof of Theorem 1.1 we see from (2.12) that in order to compute $H_{A}$ we need only those second 
partial derivatives of (3.14) that correspond to non-zero entries of $G^{-1}$. So, the derivatives we have to look at are $\partial_{s_{h}} \partial_{s_{h}} A(\mathbf{0})$, where $1 \leq h \leq r, \partial_{\mu_{i j}} \partial_{\mu_{i j}} A(\mathbf{0})$, where $1 \leq i \leq 2 r<j \leq n$, and $\partial_{\mu_{i j}} \partial_{\mu_{k \ell}} A(\mathbf{0})$, where $(i, j)$ and $(k, \ell)$ belong to the same $(p, q)$-block. First of all, $\partial_{s_{h}} \partial_{s_{h}} A(\mathbf{0})=0$ for $1 \leq h \leq r$. Second, from (3.15) and (3.17) we have that for any $1 \leq i \leq 2 r<j \leq n$

$$
\begin{aligned}
\partial_{\mu_{i j}} \partial_{\mu_{i j}} A(\mathbf{0}) & =L_{i j}^{2} \Omega-2 L_{i j} \Omega L_{i j}+\Omega L_{i j}^{2}=-L_{i j} \partial_{\mu_{i j}} A(\mathbf{0})+\partial_{\mu_{i j}} A(\mathbf{0}) L_{i j} \\
& =\omega_{\left\lfloor\frac{i+1}{2}\right\rfloor} \cdot\left\{\begin{array}{l}
-L_{i j} L_{i-1, j}+L_{i-1, j} L_{i j} \text { if } i \text { is even } \\
L_{i j} L_{i+1, j}-L_{i+1, j} L_{i j} \text { if } i \text { is odd }
\end{array}\right. \\
& =\omega_{\left\lfloor\frac{i+1}{2}\right\rfloor} \cdot\left\{\begin{array}{l}
L_{i-1, i} \text { if } i \text { is even } \\
L_{i, i+1} \text { if } i \text { is odd }
\end{array}\right.
\end{aligned}
$$

where in the second equality we used (3.23). Third, for any $(i, j)$ and $(k, \ell)$ with $(i, j) \leq(k, \ell)$ (in the lexicographic order) (3.15) and (3.17) imply that

$$
\partial_{\mu_{i j}} \partial_{\mu_{k l}} A(\mathbf{0})=L_{k \ell} L_{i j} \Omega-L_{k \ell} \Omega L_{i j}-L_{i j} \Omega L_{k \ell}+\Omega L_{i j} L_{k \ell}=-L_{k \ell} \partial_{\mu_{i j}} A(\mathbf{0})+\partial_{\mu_{i j}} A(\mathbf{0}) L_{k \ell} .
$$

Assuming that the indices $(i, j)$ and $(k, \ell)$ belong to the same $(p, q)$-group and using (3.19), (3.20), (3.21) and (3.22) we now elaborate (3.30), conveniently storing the result in a matrix,

$$
\left(\partial_{\mu_{i j}} \partial_{\mu_{k \ell}} A(\mathbf{0})\right)=\left(\begin{array}{cccc}
\overbrace{\omega_{p} L_{2 p-1,2 p}}^{+\omega_{q} L_{2 q-1,2 q}} & \overbrace{0}^{(2 p-1,2 q-1)} & \overbrace{0}^{(2 p-1,2 q)} & \overbrace{-\omega_{p} L_{2 q-1,2 q}} \\
0 & \begin{array}{c}
\omega_{q} L_{2 p-1,2 p} \\
\omega_{p} L_{2 p-1,2 p}
\end{array} & \omega_{p} L_{2 q-1,2 q} & 0 \\
+\omega_{q} L_{2 q-1,2 q} & +\omega_{q} L_{2 p-1,2 p} & 0 \\
0 & \omega_{p} L_{2 q-1,2 q} & \omega_{p} L_{2 p-1,2 p} & 0 \\
-\omega_{p} L_{2 q-1,2 q} & +\omega_{q} L_{2 p-1,2 p} & +\omega_{q} L_{2 q-1,2 q} & \omega_{p} L_{2 p-1,2 p} \\
-\omega_{q} L_{2 p-1,2 p} & 0 & 0 & +\omega_{q} L_{2 q-1,2 q}
\end{array}\right)\left\{\begin{array}{l}
(2 p, 2 q) \\
(2 p-1,2 q) \\
(2 p, 2 q-1) \\
(2 p, 2 q)
\end{array} .\right.
$$

In order to find normal components of matrices (3.29) and (3.30) we need to describe the normal space to $\delta k_{n, 2 r}$ at $A$. For this let us observe that matrices

$$
L_{a b}, \quad 2 r<a<b \leq n,
$$

are orthogonal to matrices (3.17), (3.18) that form a basis of the tangent space $T_{A} \delta k_{n, 2 r}$. Moreover, (3.32) are independent and there are $\left(\begin{array}{c}n-2 r \\ 2\end{array}\right)=\operatorname{codim}\left(\delta k_{n, 2 r}\right)$ many of them. It implies that matrices (3.32) form a basis of the normal space to $\delta k_{n, 2 r}$ at $A$. It is now elementary to check that the relevant second derivatives (3.29) and (3.31) are orthogonal to (3.32) or, equivalently, they have trivial normal components. It follows from the above reasoning that $H_{A}=0$.

In the beginning of the proof we assumed that the non-zero eigenvalues of $A \in \delta k_{n, 2 r}$ are distinct. Those $A \in \delta \AA_{n, 2 r}$ that do not satisfy this assumption form an algebraic submanifold $X \subset \delta k_{n, 2 r}$ which is proper because there are obviously matrices in $\delta k_{n, 2 r} \backslash X$. Since the mean curvature vector field $H$ is a smooth field of normal vectors to $\delta k_{n, 2 r}$ and since $H_{A}=0$ for $A$ in the open and dense subset $\delta k_{n, 2 r} \backslash X \subset \delta k_{n, 2 r}$, we have $H_{A}=0$ for all $A \in \mathcal{S} k_{n, 2 r}$.

3.3. Proof of Theorem 1.6. Our proof uses a general result of Hsiang and Lawson from HL71 which we state now. Let $(M, \boldsymbol{g})$ be a Riemannian manifold and let $G$ be a compact, connected group acting smoothly by isometries on $M$. For $x \in M$ let $G_{x}$ be the stabilizer of $x$, that is, the group of those transformations in $G$ that fix $x$. Let us consider the equivalence relation on $M$ for which points $x, y \in M$ are said to be equivalent if their stabilizers $G_{x}$ and $G_{y}$ are conjugate, that is, $g G_{x} g^{-1}=G_{y}$ for some $g \in G$. By [HL71, Sec. 1.3], equivalence classes of this relation are minimal submanifolds of $(M, \boldsymbol{g})$. 
Let us now apply this result to $(M, \boldsymbol{g})=\left(\right.$ Sym $\left._{n},\langle\cdot, \cdot\rangle\right)$ and the action (2.6) of $G=O(n)$. Recall from Subsection 2.2 that this action preserves the inner product (1.1). By Corollary 2.2. sets Sym $_{n, \vec{\kappa}} \subset$ Sym $_{n}$ are precisely the equivalence classes of the above defined equivalence relation on Sym $_{n}$ and hence they are minimal submanifolds of $\left(\right.$ Sym $\left._{n},\langle\cdot, \cdot\rangle\right)$.

\section{REFERENCES}

[Agr11] A. Agrachev, On the space of symmetric operators with multiple ground states, Functional Analysis and Its Applications 45 (2011), 241-251.

[Arn72] V. I. Arnol'd, Modes and quasimodes, Functional Analysis and Its Applications 6 (1972), 94-101.

[BKL18] P. Breiding, Kh. Kozhasov, and A. Lerario, On the geometry of the set of symmetric matrices with repeated eigenvalues, Arnold Math J. 4 (2018), 423-443.

[FL83] W. Fulton and R. Lazarsfeld, Positive polynomials for ample vector bundles, Annals of Mathematics 118 (1983), no. 1, 35-60.

[Har92] J. Harris, Determinantal varieties, Algebraic Geometry. Graduate Texts in Mathematics, vol. 133, Springer, New York, NY, 1992.

[HL71] W.-Y. Hsiang and H. B. Lawson, Jr., Minimal submanifolds of low cohomogeneity, J. Differential Geom. 5 (1971), no. 1-2, 1-38.

[HLT17] J. Hoppe, G. Linardopoulos, and O. T. Turgut, New minimal hypersurfaces in $\mathbb{R}^{(k+1)(2 k+1)}$ and $S^{(2 k+3) k}$, Math. Nachr. 290 (2017), 2874-2878.

[Hop19] J. Hoppe, Lectures on minimal surfaces, arXiv:1903.12062 [math.DG], 2019.

[Hsi67] W-Y. Hsiang, Remarks on closed minimal submanifolds in the standard Riemannian m-sphere, J. Differential Geom. 1 (1967), no. 3-4, 257-267.

[KN69] S. Kobayashi and K. Nomizu, Foundations of Differential Geometry, vol. 2, Interscience publishers, 1969.

[TF87] D. T. Thi and A. T. Fomenko, Minimal surfaces, stratified multivarifolds, and the Plateau problem, "Nauka", Moscow, 1987.

[Tho88] G. Thompson, Normal forms for skew-symmetric matrices and Hamiltonian systems with first integrals linear in momenta, Proc. Amer. Math. Soc. 104 (1988), 910-916.

[Tka10] V. Tkachev, Minimal cubic cones via Clifford algebras, Complex Anal. Oper. Theory 4 (2010), $685-700$.

Khazhgali Kozhasov, Technische Universität Braunschweig, Institut Für Analysis und Algebra, Universitätsplatz 2, 38106 Braunschweig, Germany

E-mail address: k.kozhasov@tu-braunschweig.de 